Received: 19 October 2009, Accepted: 29 September 2010

Edited by: A. G. Green

Reviewed by: T. Ehrhardt, Math. Dept., Univ. California, Santa Cruz, USA

Licence: Creative Commons Attribution 3.0

DOI: 10.4279 /PIP.020003

www.papersinphysics.org

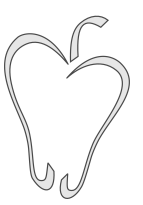

ISSN 1852-4249

\title{
Expansions for eigenfunction and eigenvalues of large- $n$ Toeplitz matrices
}

\author{
Leo P. Kadanoff ${ }^{1,2 *}$
}

\begin{abstract}
This paper constructs methods for finding convergent expansions for eigenvectors and eigenvalues of large- $n$ Toeplitz matrices based on a situation in which the analogous infinite$n$ matrix would be singular. It builds upon work done by Dai, Geary, and Kadanoff $[\mathrm{H}$ Dai et al., J. Stat. Mech. P05012 (2009)] on exact eigenfunctions for Toeplitz operators which are infinite-dimension Toeplitz matrices. One expansion for the finite- $n$ case is derived from the operator eigenvalue equations obtained by continuing the finite- $n$ Toeplitz matrix to plus infinity. A second expansion is obtained by continuing the finite- $n$ matrix to minus infinity. The two expansions work together to give an apparently convergent expansion for the finite- $n$ eigenvalues and eigenvectors, based upon a solvability condition for determining eigenvalues. The expansions involve an expansion parameter expressed as an inverse power of $n$. A variational principle is developed, which gives an approximate expression for determining eigenvalues. The lowest order asymptotics for eigenvalues and eigenvectors agree with the earlier work [H Dai et al., J. Stat. Mech. P05012 (2009)]. The eigenvalues have a $(\ln n) / n$ term as their leading finite- $n$ correction in the central region of the spectrum. The $1 / n$ correction in this region is obtained here for the first time.
\end{abstract}

\section{Introduction}

\section{i. History}

This paper is a continuation of recent work by Dai, Geary, and Kadanoff [1] (which we shall hereafter cite as paper I) and Lee, Dai and Bettleheim [2] on the spectrum of eigenvalues and eigenfunctions for singular Toeplitz matrices. A Toeplitz matrix is one in which the matrix elements, $T_{j, k}$, are functions of the difference between indices. We define all matrix elements in terms of a single function: the symbol, $a(z)$, where $z=e^{-i p}$ is on the unit circle. Thus we

\footnotetext{
*E-mail: lkadanoff@gmail.com

1 The Perimeter Institute, Waterloo, Ontario, Canada.

2 The James Franck Institute, The University of Chicago, Chicago, IL USA.
}

write

$$
T_{j, k}=T_{j-k}=\oint \frac{d z}{2 \pi i z} \frac{a(z)}{z^{j-k}}
$$

The Toeplitz matrix is then defined by having the indices $j$ and $k$ live in the interval [0,n-1]. (Note that I use the subscript notation to describe behavior in coordinate space, and argument notation to describe behavior in Fourier space.)

The basic problem under consideration here is the definition of a good method for calculating the eigenvalues and eigenfunctions of Toeplitz matrices for large values of $n$. Previous work [3, 4] has described the Toepltiz matrix problem by pointing out that the eigenvalues approach the spectrum of the analogous problem in which the indices vary over the set $[-\infty, \infty]$. This latter problem may be solved by Fourier transformation and has an eigenfunction $\Psi_{j}=e^{-i p j}$ and a corresponding eigenvalue $a\left(e^{-i p}\right)$. The set of all such eigenvalues, for real $p$, is termed image of the symbol. Widom speculates 
$[3,4]$ that in the large- $n$ limit, the discrete spectrum of the finite- $n$ problem approaches that image, at least for the case in which the symbol has a singularity on the unit circle.

Previous work $[2,1]$ has established how this approach occurs for the specific case in which the symbol has the form of singularity introduced by Fisher and Hartwig [9, 10], specifically

$$
a(z)=(2-z-1 / z)^{\alpha}(-z)^{\beta}
$$

Note that this singularity is defined by two parameters, $\alpha$, which defines a zero in the symbol and, $\beta$, which defines a discontinuity. For this symbol, Lee, Dai, and Bettleheim [2] found the spectrum for large $n$ and $\alpha=0$, while Dai, Geary and Kadanoff described a part of the spectrum for real parameters, $\alpha$ and $\beta$, obeying $0<\alpha<|\beta|<1$ in paper I. The spectrum of the Toeplitz matrix is invariant under a reflection, $\beta \rightarrow-\beta$, in the sign of $\beta$.

Paper I considered the behavior of Toeplitz operators $[7,8]$ constructed from the symbol of Fisher and Hartwig. These are Toeplitz matrices in which the indices run through the interval $[0, \infty]$. The analysis was carried on for situations in which $0<\alpha<1$ so that the image of the symbol forms a closed curve. The finite- $n$ eigenvalues sit within that curve and approach it as $n$ goes to infinity. Two cases should be differentiated:

Case I. $0>\beta>-1$. All points within the image of the symbol are right eigenvalues of the Toeplitz operator [1].

Case II. $0<\beta<1$. The Toeplitz operator has no right eigenvalues [1].

(There is one more very interesting special case: $\alpha=0$. In this situation, if $-1<\beta<1$, the image of the symbol is a curved line segment, and the eigenvalue spectrum consists of all points which can be reached by connecting two points of that curve. Once again, the eigenvalues for finite- $n$ approach the curve while sitting within the region defined by the infinite- $n$ eigenvalues [2]. We do not consider this $\alpha=0$ case in this paper.)

The distinction between cases I and II above describes whether or not the Toeplitz operator has or has not right eigenvectors. The transposition operation,

$$
T_{j, k}=T_{j-k} \rightarrow T_{k, j}=T_{k-j}
$$

is just the parity operation on $j-k$, and can be represented by flipping the sign of $\beta$ in the symbol.
Thus if $T$ is in the category of case I, its transpose is in case II - and vice versa.

This distinction carries over in a subtle manner to the Toeplitz matrices. In case I, the right eigenvectors, $\Psi_{j}$ for the Toeplitz matrices decay exponentially as $j$ increases. The corresponding left eigenvectors for the operator with the same (negative) value of $\beta$ grow exponentially with increasing $j$. This growth can be seen from an additional symmetry of the Fisher-Hartwig Toeplitz matrix under the reflection operation that changes the index value, $j$, into $n-1-j$.

$$
T_{j, k}=T_{j-k} \rightarrow T_{(n-1-j)-(n-1-k)}=T_{k-j}
$$

and has the same effect as the transposition operation. The reflection interchange flips the sign of $\beta$ and also makes the decay of the right eigenvector with $j$ into a growth with $j$. Thus, the case I-case II distinction is interchanged for both Toeplitz operator and Toeplitz matrix under the transposition symmetry, and is equally interchanged for the matrix under the reflection operation.

\section{ii. The previous calculational strategy}

In the previous paper, paper I, we studied the Toeplitz eigenvalue equation for case I

$$
\sum_{k=0}^{n-1} T_{j-k} \psi_{k}=\epsilon \psi_{j} \text { for } 0 \leq j \leq n-1
$$

by studying the related Toeplitz operator equation

$$
\sum_{k=0}^{\infty} T_{j-k} \Psi_{k}=\epsilon \Psi_{j} \text { for } 0 \leq j \leq \infty
$$

for an eigenvalue for which both equations equally had solutions. We could only solve the first equation numerically. We had an exact method, the Wiener-Hopf technique, for solving the second equation. The crucial result was that, for case I situations and for large $n$, the solution of the second equation provided an excellent approximation for the eigenfunction of the first one, at least in the situation in which one is given the correct eigenvalue. What happened was that the extension of the equation being solved into the region between $j=n$ and $j=\infty$ hardly changed the solution of Eq. (5a), at least for $j$ not too close to $n$. 
The next step will be to study an equation arising from extending Eq. (5a) toward minus infinity, specifically

$$
\sum_{k=-\infty}^{n-1} T_{j-k} \Omega_{k}=\epsilon \Omega_{j}+\Gamma_{j} \text { for }-\infty \leq j \leq n-1
$$

Notice the forcing term, $\Gamma$, on the right hand side of this equation. In the same case I, situation in which Eq. (5b) has eigenvalue solutions, Eq. (5c) has none so that the forcing term produces a unique, finite result. Very similar methods to the ones which solve Eq. (5b) will also solve Eq. (5c).

\section{iii. Plan of paper}

Roughly speaking, the plan of this paper is to produce and combine two different expansions for the Toeplitz matrix equation. First, one will get an approximate solution to Eq. (5b), albeit with some small terms left over. Next, the methods used to solve Eq. (5c) will be used to calculate these leftover terms while treating the terms previously determined as forcings. We shall thereby close the equations for the Toeplitz matrix eigenvector.

The previous work, paper I, had a rather heuristic method for estimating the size of the corrections to the eigenvalue and eigenfunction estimates. Here we have an exact, testable expansion. However, the expansion does start from the premise that the finite- $n$ spectrum of eigenvalues does approach the infinite- $n$ spectrum, a premise that is true for a wide class of Toeplitz matrices with singular symbols $[5,6]$.

The next chapter includes the two analyses respectively based upon the two Toeplitz operator equations obtained by extending our matrix in the two possible directions. The third chapter puts the two analyses together to get equations which will yield an asymptotic expansion for eigenvalues and eigenfunctions. The final chapter describes Toeplitz problems left unresolved by this paper.

\section{A pair of expansions}

\section{i. Definitions}

In all three cases defined by Eqs. (5), our analysis will be generated by extending the range of the index variables to $(-\infty, \infty)$, which will then permit us to use Fourier transform techniques. The equation for the Toeplitz matrix's eigenvector can be cast in terms of three different kinds of functions which are respectively indicated by superscripts -, 0 , and + . The first superscript indicates a function which is non-zero only for $j<0$; the superscript 0 defines a function non-zero for $0 \leq j \leq n-1$; while the third superscript describes a function non-zero in $[n, \infty)$. The eigenfunction we wish to calculate is $\psi^{0}{ }_{j}$ and it obeys

$$
\sum_{k=-\infty}^{\infty} K_{j, k} \psi_{k}^{0}=\phi^{-}{ }_{j}+\phi^{+}{ }_{j},
$$

which holds for all integer values of $j$. Here, the matrix $K$ is

$$
K_{j, k}=T_{j-k}-\epsilon \delta_{j, k}
$$

Eq. (6) will be analyzed in Fourier transform language, with $z$ being the Fourier variable, as in Eq. (1). Thus, the four quantities defined in that equation will be written as $K(z)=a(z)-$ $\epsilon, \phi^{-}(z), \psi^{0}(z)$, and $\phi^{+}(z)$, which will respectively contain powers of $z$ extending from $-\infty$ to $\infty$; only negative powers of $z$; non-negative powers extending up to $z^{n-1}$; and powers from $z^{n}$ to $z^{\infty}$. We also need to define a notation for the decomposition of the $K$ operator. We write, for case I, the Wiener-Hopf factorization

$$
K(z)=K^{>}(z) /\left(z K^{<}(z)\right)
$$

where $K^{>}$has all its singularities and zeros outside the unit circle and $K^{<}$has all its singularities and zeros inside the unit circle. The reader should recall, from paper I, if and only if $\epsilon$ is inside the curve described by a (z). (For case II, the $z$ would appear in the numerator rather than the denominator.) The functions $K^{>}(z)$ and $1 / K^{>}(z)$ have neither zero nor singularity inside the unit circle so that they can be expanded in a power series in $z$. Similarly, $K^{>}(z)$ and $1 / K^{>}(z)$ are regular outside the unit circle so that they can be expanded in $1 / z$. As a result, the Fourier transforms of these functions obey

$$
K^{>}{ }_{j-l}=0 \text { for } j<l
$$

while 


$$
K^{<-l}=0 \text { for } l<j .
$$

One can equally well define the functions $\left(1 / K^{>}\right)$ and $\left(1 / K^{<}\right)$in coordinate space by Fourier transformation, as for example,

$$
\left(1 / K^{>}\right)_{j}=\oint \frac{d z}{2 \pi i z} z^{-j} / K^{>}(z)
$$

Acting to the right, $K^{>},\left(1 / K^{>}\right)$and $z$ all carry information toward larger $j$ values, while $K^{<}$, $\left(1 / K^{<}\right)$and $1 / z$ carry information toward lower $j$ values.

\section{ii. Wiener-Hopf analysis for Toeplitz oper- ator}

This section is not at all new. It is all contained in paper I and in earlier work $[7,8]$. However, the notation is slightly different here. We set $n=\infty$ and note that $\phi^{+}$must be zero. To distinguish the solution for the Toeplitz operator from the one for the Toeplitz matrix, we write $\Psi$ for the operator eigenfunction and $\Phi^{-}$for the auxiliary function $\phi^{-}$. We then note that Eqs. (5b), (7) and (8) imply

$$
K^{>} \Psi=z K^{<} \Phi^{-}
$$

Note that $\Psi$ contains only non-negative powers of $z$, while $\Phi^{-}$contains only negative powers.

Eq. (9) is constructed to enable us to follow the usual Wiener-Hopf strategy [11]. The only possible common behavior of the two sides of Eq. (9) is that both sides may contain a constant term, independent of $z$. Then Eq. (9) has the solution

$$
\begin{gathered}
K^{>} \Psi=C \\
z K^{<} \Phi^{-}=C
\end{gathered}
$$

with $C$ being simply an arbitrary constant in this Fourier transform language. (In coordinate space, $C$ becomes $C \delta(j, 0))$. The solution can then be written in terms of two functions:

$$
\Psi_{j}=C\left(1 / K^{>}\right)_{j}
$$

which vanishes for $j<0$, while the other function is

$$
\begin{array}{r}
\Phi_{j}^{-}=C\left(1 / K^{<}\right)_{j+1} \\
\text { with }\left(1 / K^{<}\right)_{j}=\oint \frac{d z}{2 \pi i z} z^{-j} / K^{<}(z) .
\end{array}
$$

This integral vanishes for $j>0$. Note that the arbitrary parameter, $C$, is a normalization constant for the eigenfunction and its auxiliary function, $\Phi^{-}$.

The analysis in paper I enables us to describe the asymptotic structure of these functions for values of $|j|$ much bigger than one in the previously analyzed case $0<\alpha<-\beta<1$. Recall from paper I that $\epsilon=a\left(z_{c}\right)$, that $z_{c}=e^{-i p_{c}}$ is outside the unit circle, and therefore $\epsilon$ is inside the curve formed by $a(z)$, with $z$ on the unit circle. The Fourier transforms of both functions contain a weak singularity at $z=1$ proportional to $(1-z)^{2 \alpha}$. This zero, then, produces a real term which decays as $1 / j^{1+2 \alpha}$ for large values of $|j|$. The function, $K^{>}(z)$, has, in addition to the weak zero, a simple zero at $z=z_{c}$, just outside the unit circle. This zero describes the eigenvalue of the Toeplitz matrix. The zeros give an asymptotic form for large $j$ containing two terms

$$
\left(1 / K^{>}\right)_{j} \rightarrow A^{>}\left(e^{-i p_{c}}\right) j^{-1-2 \alpha}+B\left(e^{-i p_{c}}\right) e^{i p_{c} j},
$$

The notation, $A^{>}\left(e^{-i p_{c}}\right)$ and $B\left(e^{-i p_{c}}\right)$, indicates that these coefficients depend upon the eigenvalue. For $\left(1 / K^{<}\right)$and thus for the auxiliary function, $\Phi^{<}{ }_{j}$, this singularity defines the behavior for large values of $-j$ as

$$
\left(1 / K^{<}\right) \rightarrow A^{<}\left(e^{-i p_{c}}\right)(-j)^{-1-2 \alpha}
$$

Thus, $\Psi_{j}$ and $\Phi_{-j}$ both decay algebraically for large values of $j$.

Paper I suggested that the two terms on the right-hand-side of Eq. (12a) were both of order $n^{-1-2 \alpha}$ when $j$ is of order $n$. This result gives us a small parameter

$$
\lambda \sim n^{-1-2 \alpha} \sim\left|e^{i p_{c} n}\right|
$$

which might be used in expansions.

\section{iii. Approximate eigenvector for Toeplitz matrix}

Equation 6 can be analyzed using the same kinds of splitting of $K$ employed in Sec. ii. We take that equation in its Fourier transformed representation, namely,

$$
K(z) \psi^{0}(z)=\phi^{+}(z)+\phi^{-}(z)
$$

and multiply by $z K^{<}$as in Eq. (9). One then finds

$$
K^{>} \psi^{0}=z K^{<} \phi^{-}+z K^{<} \phi^{+}
$$


Papers in Physics, vol. 2, ART. 020003 (2010) / L. P. Kadanoff

The second term on the right hand side of this equation can be split up into parts which contain exponents of $z$ which are respectively negative, between zero and $n-1$ inclusive, and above $n-1$ in the form

$$
z K^{<} \phi^{+}=\left(z K^{<} \phi^{+}\right)^{-}+\left(z K^{<} \phi^{+}\right)^{0}+\left(z K^{<} \phi^{+}\right)^{+}
$$

Then this equation can be rearranged into a form in which terms in non-positive powers of $z$ appear on one side and non-negative powers on the other. i.e.

$$
\begin{array}{r}
K^{>} \psi^{0}-\left(z K^{<} \phi^{+}\right)^{0}-\left(z K^{<} \phi^{+}\right)^{+} \\
=z K^{<} \phi^{-}+\left(z K^{<} \phi^{+}\right)^{-}=c
\end{array}
$$

The second equal sign in this equation sets both sides equal to a constant, $c$, as in Eq. (10). The leading terms in both the wave function and the auxiliary function, $\phi^{-}$, are set by $c$ since Eq. (16) implies a leading order behavior

$$
K^{>}{ }_{0} \psi^{0}=K_{0}^{<_{0}} \phi^{-}{ }_{-1}=c
$$

for $j$ 's not too far from zero. Later on, we shall use an analogous result from an analysis of forcings in Eq. (14) to obtain a solvability equation for determining the eigenvalues. Notice that we have used the symbol $c$ to describe the normalization constant in this situation, while we used $C$ for the same purpose in the Toeplitz-matrix eigenvector. These two quantities are analogous, but need not be the same.

We solve for $\psi^{0}$, finding

$$
\begin{aligned}
\psi^{0}=\left(1 / K^{>}\right) c & +\left(1 / K^{>}\right)\left(z K^{<} \phi^{+}\right)^{0} \\
+ & \left(1 / K^{>}\right)\left(z K^{<} \phi^{+}\right)^{+} .
\end{aligned}
$$

Since $\left(1 / K^{>}\right)$, acting to the right pushes coordinate indices toward higher values, we can project onto the subspace 0 -note that the projection of the final term is zero - and thus find

$$
\psi^{0}=\left[\left(1 / K^{>}\right) c\right]^{0}+\left[\left(1 / K^{>}\right)\left(z K^{<} \phi^{+}\right)^{0}\right]^{0}
$$

The equation for the negative $j$ domain in Eq. (16) may be solved for $\phi^{-}$to give

$$
\phi^{-}=\left(z K^{<}\right)^{-1} c-\left(z K^{<}\right)^{-1}\left(z K^{<} \phi^{+}\right)^{-}
$$

As in the analysis in Subsection ii. the auxiliary function $\phi^{-}$does not directly enter the analysis of the eigenfunction, which is there denoted as $\Psi$, and is here called $\psi^{0}$. However, there are important differences between the result here and the one in Subsection ii.: In contrast to the case of the Toeplitz operator, the solution for the eigenfunction requires a knowledge of a subsidiary function, here $\phi^{+}$. This function provides the forcing term which renders our lowest order solution inexact. Further, for the Toeplitz operator, only the function $K^{>}$is needed to determine the eigenfunction. Here, both $K^{>}$and $K^{<}$are involved.

Note the $1 / z$ to the right of the equal sign in Eq. (18b). This factor has the effect of making the leading term in the expansion of $\phi^{-}$be $1 / z$, which is then followed by higher powers of $1 / z$. This is precisely the right structure for the expansion of $\phi^{-}$.

Eq. (18) gives us expressions for two of the quantities we need to know. However, we are far from done. These equations give us relatively simple expressions for $\psi^{0}$ and $\phi^{-}$, but we do not yet have an equivalently simple expression for $\phi^{+}$. In both of the two subequations in Eq. (18), we can evaluate the first term directly, while the second term could be evaluated by quadratures if we but knew $\phi^{+}$. Note that the first terms on the right in both of these subequations are precisely the same as in the solution for the Toeplitz operator eigenvector.

Our previous results [1] show that for small and intermediate values of $j$ in the set $[0, n-1]$, the first term in Eq. (18a) varies over a wide range, being of order $c$ for small values of $j$ and of order $c \lambda \sim$ $c / n^{2 \alpha+1}$ for $j$ of order $n$. Similarly the first term in Eq. (18b) varies from being of order $c$ for $-j$ of order unity to being of order $c \lambda$ for $-j$ of order $n$. It will turn out that the second term in each of these equations is a correction of order $c \lambda$ and therefore smaller by a factor of $\lambda$ than the maximum value of the first term.

\section{iv. Forcing analysis for Toeplitz matrix}

It appears that we have usable lowest order results for two of the three unknown functions. The third unknown, $\phi^{+}$, contributes correction terms but it is hard to see a direct way to get it from Eq. (16). However, we can use the forcing form of the Toeplitz matrix to obtain additional information. To do this, rewrite Eq. (15) while interchanging 
the role of $K^{>}$and $K^{<}$and find

$$
\left(1 / K^{<}\right) \psi^{0}=\left(z / K^{>}\right) \phi^{-}+\left(z / K^{>}\right) \phi^{+}
$$

We may then split up the term $\left(z / K^{<}\right) \phi^{-}$into the different regions $(-, 0$, and + ) to derive a result analogous to Eq. (16), namely

$$
\begin{array}{r}
\left(1 / K^{<}\right) \psi^{0}-\left[\left(z / K^{>}\right) \phi^{-}\right]^{0}-\left[\left(z / K^{>}\right) \phi^{-}\right]^{-} \\
=\left(z / K^{>}\right) \phi^{+}+\left[\left(z / K^{>}\right) \phi^{-}\right]^{+}=0
\end{array}
$$

There is, however, a substantial difference between Eq. (16) and Eq. (20). Look at the region to the right of the first equal sign in both equations. The former has a $z \phi^{-}$in it, while the latter has a $z \phi^{+}$. The former has a component which extends beyond the region - into the region 0 , while the latter has no term projecting our of the region + . Thus, we put a constant, $c$, on the right hand side of Eq. (16) to cancel the extending term, but put a zero on the right of Eq. (20), since there is no such term. This zero is, at bottom, a reflection of the fact that the transposed Wiener-Hopf operator equation has no eigenvalue solutions.

Now look at the terms between the two equal signs in Eq. (20). The lowest power of $z$ in the first such term is $z^{n+1}$. The next term contains as its smallest power of $z$, a term in $z^{n}$. There is nothing to balance this term. It must vanish. It follows that

$$
\left[\left(z / K^{>}\right) \phi^{-}\right]_{n}=0
$$

This statement will turn out to be the integrability condition that will fix the eigenvalue in our analysis.

We now use Eq. (20) to write the analogs of Eq. (18a) and Eq. (18b), which are

$$
\psi^{0}=\left[K^{<}\left(\left(z / K^{>}\right) \phi^{-}\right)^{0}\right]^{0}
$$

and

$$
\phi^{+}=-\left(K^{>} / z\right)\left[\left(z / K^{>}\right) \phi^{-}\right]^{+}
$$

Eq. (22a) is another expression for the eigenfunction, analogous to Eq. (18a). We hope that the two equations are equivalent. Eq. (22b) gives us a usable expression for $\phi^{+}$, which can then be employed to give explicit values to the correction terms in Eq. (18a) and Eq. (18b). Note that Eq. (22a) and Eq. (22b) are simpler than their analogs, derived earlier, because they do not have terms in $c$. These four equations will give us the results we need for the various eigenfunctions. They are exact; there are no approximations made in their derivation. The integrability condition, Eq. (21), is also exact.

\section{Results}

\section{i. Estimation of eigenvalues}

The eigenvalue of the Toeplitz matrix can be estimated with the help of Eq. (21). When written out, this equation reads

$$
\sum_{j=1}^{\infty}\left(1 / K^{>}\right)_{n+j-1} \phi_{-j}^{-}=0
$$

To obtain a lowest order version of this equation, one replaces $\phi^{-}$by its lowest order approximant, as given by the first term on the right hand side of Eq. (18b). We thereby obtain

$$
\sum_{j=1}^{\infty}\left(1 / K^{>}\right)_{n+j-1} K^{<}{ }_{1-j}=0
$$

as our lowest order eigenvalue condition.

In the situation in which $n$ is large, one can use the asymptotic form of $\left(1 / K^{>}\right)$as given by Eq. (12a) to replace the first factor under the summation in Eq. (24) so that the eigenvalue condition becomes

$$
\begin{gathered}
\sum_{j=1}^{\infty}\left[A^{>}\left(e^{-i p_{c}}\right)(n+j-1)^{-1-2 \alpha}\right. \\
\left.+B\left(e^{-i p_{c}}\right) e^{i p_{c}(j-1)} e^{i p_{c} n}\right] K_{1-j}^{<}=0 .
\end{gathered}
$$

The main contribution to this equation converges rapidly with $j$, so for large $n$ we neglect $j$ in comparison to $n$ and find an expression for the momentumvalue, $p_{c}$ :

$$
e^{i p_{c} n}=-n^{-1-2 \alpha} \frac{\left[A^{>}\left(e^{-i p_{c}}\right) \sum_{j=1}^{\infty} K_{1-j}^{<}\right]}{\left[B\left(e^{-i p_{c}}\right) \sum_{k=1}^{\infty} e^{i p_{c}(k-1)} K_{1-k}^{<}\right]}
$$

This equation is then solved to get an asymptotic expansion for the $m$-th value of the momentum 


$$
\begin{aligned}
p_{c}^{(m)} & =2 \pi(-1+m / n)-(1+2 \alpha) i(\ln n) / n \\
& +(\delta p) i / n+o(1 / n), \\
\text { with }(\delta p) & =\ln \left\{\left[-A^{>}\left(z_{c}\right) K^{<}(1)\right] /\left[B\left(z_{c}\right) K^{<}\left(z_{c}\right)\right]\right\}, \\
\text { and } z_{c} & =e^{-i p_{c}}
\end{aligned}
$$

Here, $m$ is a label for the different eigenvalues, which takes on values between zero and $n-1$. In Eq. (25), $A^{>}$and $B$ are non-singular functions of $m / n$, except for extra corrections which appear when $m$ is close to its endpoints. Thus, $\delta p$ gives smaller, slowing varying corrections to the earlier terms on the right of Eq. (25). The estimates reflected in Eq. (25) were all predicted in paper I, except for the precise value of $\delta p$, which appears here for the first time.

\section{ii. Equations for eigenfunctions}

We argue about the relative sizes of the various terms in the equations by saying that $1 / K^{>}$and $1 / K^{<}$serve as propagators which connect the regions described by the symbols,- 0 , and + . Any connection between - and + is necessarily small, as is any connection of $c \delta_{j, 0}$ to + . I assert that these connections are of order $\lambda \sim 1 / n^{1+2 \alpha}$. This smallness makes terms involving several regions necessarily small and makes it possible for our expansions for the eigenfunction and auxiliaries, given below, to be rapidly convergent.

To obtain such expansions, one starts with the unknown that determines $\phi^{-}$, as seen in Eq. (18b), i.e.

$$
X^{-}=\left(z K^{<} \phi^{+}\right)^{-}
$$

According to the arguments we have given so far, this term should be at maximum of order $c \lambda^{2}$. Then rewrite Eq. (22b) for $\phi^{+}$in terms of this small unknown quantity as

$$
\phi^{+}=-\left(K^{>} / z\right)\left[\left[1 /\left(K^{>} K^{<}\right)\right]\left[c-X^{-}\right]\right]^{+}
$$

We can next rewrite this equation as in integral equation for the unknown as

$$
X^{-}=-\left(\left(K^{>} K^{<}\right)\left[\left(K^{>} K^{<}\right)^{-1}\left(c-X^{-}\right)\right]^{+}\right)^{-}
$$

Since $X^{-}$is of order $\lambda^{2}$ relative to $c$, Eq. (28) can be solved iteratively by expanding the right hand side in a power series in $X^{-}$.
Once $X^{-}$is determined, Eq. (18b) will give the value of $\phi^{-}$and Eq. (27) will determine $\phi^{+}$. Using the value of $\phi^{+}$one can then determine the eigenfunction via Eq. (18a).

\section{iii. An unconventional eigenvalue condition}

It appears that we have a convergent expansion for our eigenfunction. However, there is a potential difficulty. The expansions cannot always converge. Indeed, the equations for the various functions determine an eigenvalue, and cannot possibly converge unless the eigenvalue condition is met. The reader might not be sure that the integrability condition of Eq. (21) is the correct eigenvalue condition.

One conventional way of finding eigenvalues is through the use of an extremal principle. Such a principle always exists for a Hermetian matrix. The matrix, $T$, is not Hermetian, but, as a Toeplitz matrix, it has a built-in reflection symmetry which can be used in a roughly similar manner. Let $\chi_{j}$ be a vector with indices, $j$ 's, in the interval $[0, n-1]$. Then, the reflection of this eigenvector is

$$
\tilde{\chi}_{j}=\chi_{n-1-j}
$$

As discussed in paper I, if $\chi$ is an eigenvector of the this Toeplitz matrix, $T$, then the reflected vector is automatically an eigenvector of the transpose of $T$. In symbols

$$
\sum_{k=0}^{n-1} K_{j-k} \chi_{k}=0 \text { implies } \sum_{k=0}^{n-1} \chi_{n-1-k} K_{k-\tilde{j}}=0
$$

where $\tilde{j}=n-1-j$. Thus, if the left-hand statement is true for all $j$ in $[0, n-1]$, then the right-hand statement is equally true.

Now we define an analog of an extremal principle for the Toeplitz matrix. Consider the quantity:

$$
\begin{aligned}
Q[\chi] & =N / D \text { with } \\
D & =\sum_{l=0}^{n-1} \tilde{\chi}_{l} \chi_{l}=\tilde{\chi} \cdot \chi \\
N & =\sum_{j, k=0}^{n-1} \tilde{\chi}_{j}\left[T_{j-k}-\epsilon \delta_{j, k}\right] \chi_{k}=[\tilde{\chi} \cdot K \cdot \chi]
\end{aligned}
$$

This quantity, $Q$, reduces to zero when $\epsilon$ is an eigenvalue of the $n$-th order Toeplitz matrix and $\chi$ is 
the corresponding eigenfunction. If $\chi$ deviates from this eigenfunction by a small amount, then $Q$ is of order of the square of the deviation. If we choose a variational function that is an eigenfunction, but one with the "wrong" eigenvalue, the variational function will have the value of the difference between $\epsilon$ and that eigenvalue. Since the eigenvalues vary by an amount of order unity, we might expect that a completeness argument might imply that for an arbitrarily chosen smoothly varying $\chi, Q$ would be of order unity.

Of course, if $\epsilon$ is not an eigenvalue of the Toeplitz matrix, this extremal property is lost. Unfortunately, the extremal property is not a minimum or a maximum property. Hence, it might be that an incorrect variational function would nonetheless give the variational function a value zero.

If $Q$ is far from zero, $\chi$ deviates considerably from the eigenvector. However, the converse is not true: $Q$ might be zero, while $\chi$ is nonetheless far from being an eigenvector. However, we shall use a small size of $Q$ as some indication of a good approximation to an eigenvector.

Now look at the special case in which $\chi$ is our lowest order approximation for the eigenfunction as given by the first term in Eq. (18a),

$$
\chi_{j}=\Psi_{j}=\left(1 / K^{>}\right)(j) \text { for } 0 \leq j \leq n-1
$$

(For simplicity, we have chosen $C=1$.) With this choice the denominator has the value

$$
D=\sum_{l=0}^{n-1}\left(1 / K^{>}\right)(l)\left(1 / K^{>}\right)(n-1-l)
$$

The first step in simplifying the numerator is to replace the sum over $k=[0, n-1]$ by a sum over $k=[0, \infty]$ minus a sum over $k=[n, \infty]$. The sum over $[0, \infty]$ vanishes so that

$$
N=-\sum_{j=0}^{n-1} \sum_{k=n}^{\infty} \Psi_{n-1-j} \Psi_{k} K_{j-k}
$$

The $j$-sum is split into pieces. We sum over $j=$ $[0, \infty]$ and subtract the piece $j=[n, \infty]$. Eq. (9) then gives a result in which $N$ comes out as the sum of two terms, $N=N^{++}+N^{+-}$which respectively have the values

$$
\begin{aligned}
N^{++} & =\sum_{j, k=0}^{\infty} \Psi_{j+n} \Psi_{k+n} K_{-j-k-1-n} \\
& =\sum_{j, k=0}^{\infty}\left(1 / K^{>}\right)_{j+n}\left(1 / K^{>}\right)_{k+n} K_{-j-k-1-n}
\end{aligned}
$$

and

$$
\begin{aligned}
N^{+-} & =-\sum_{k=0}^{\infty} \Psi_{k+n} \Phi_{-k-1} \\
& =-\sum_{k=0}^{\infty}\left(1 / K^{>}\right)_{k+n}\left(1 / K^{<}\right)_{-k-1}
\end{aligned}
$$

The results of paper I enable us to estimate the order of magnitude of the various terms in Eq. (33). The denominator Eq. (33a) has the magnitude $D=O(n \lambda)$, unless it is made smaller by a cancellation in the sum. The numerator term $N^{++}$has the order of magnitude $N^{++}=O\left(n \lambda^{3}\right)$ since each $K$ in the product is of order $n \lambda$ and the sum converges by falling off algebraically. The numerator term $N^{+-}$has the order of magnitude $N^{+-}=O(\lambda)$ since $\left(K^{<}\right)$falls off quite rapidly from its values, of order 1 , for small values of $k$. In fact, we already calculated precisely this term when we gave our lowest order equation for the eigenvalue in Eq. (24) derived from it our lowest order estimate estimate for $p_{c}$ in Eq. (25). Thus, our lowest order estimate was a demand that $N^{+-}$vanish. If we use the condition $N^{++}+N^{+-}=0$ to obtain another estimate of $p_{c}$, that estimate may be expected to be of higher order in $\lambda$ than the previous one. Thus, we gain additional confidence that our previous analysis is correct.

In principle, we could carry out our expansions of the eigenfunction and auxiliary functions to any derived order and thereby make our presumed exact condition of Eq. (21) true to any order. Our result for the eigenvalue is "exact", but it is not rigorous since we have not proved that our approach converges.

\section{Looking forward}

We have now completed our task of constructing an analytic (albeit heuristic) structure for an eigenfunction expansion. The work is plausible but not 
proven. The next step might be to construct proofs of the convergence and exactness of these results, or alternatively, to back them up with good numerical work.

The work, in fact, lacks two checks which one might hope to put into place using purely analytic means. I have not checked that the analytics yields the Fisher and Hartwig $[9,10]$ results for the product of eigenvalues. In fact, I cannot see from where they might arise. I also have not checked that somewhat different approaches of Subsection iii. and Subsection iv. give exactly the same expression for the eigenvector.

In addition, I don't know how the eigenvectors and eigenvalues at the ends of the spectrum behave. As shown by numerical evidence, they behave differently from the ones at the middle of the spectrum, but the difference has been left unexplored up to now. The difference arises because there are two different zeros in $K(z)$ that are well separated in the middle of the spectrum, but come together at the ends. But those words do not tell us the answer without further work.

Of course, there is much room for analysis of further regions of the parameters $\alpha$ and $\beta$.

It is, in some respects, very pleasing to see that there is yet room for good additional work on this problem.

\section{Acknowledgment}

I appreciate the help given to me by Michael Fisher and Jacques H.H. Perk. I had useful conversations with Peter Constantin, Hui Dai and Seung Yeop Lee. This work was completed during a visit to the Perimeter Institute, which is supported by the Government of Canada through Industry Canada and by the Province of Ontario through the Ministry of Research and Innovation. This work was also supported in part by the University of Chicago MRSEC program under NSF grant number DMR0213745.
[1] H Dai, Z Geary, L P Kadanoff, Asymptotics of eigenvalues and eigenvectors of Toeplitz matrices, J. Stat. Mech. P05012 (2009).

[2] S Y Lee, H Dai, E Bettelheim, Asymptotic eigenvalue distribution of large Toeplitz matrices, arXiv:0708.3124v1 (2007).

[3] H Widom, Toeplitz determinants with singular generating functions, Amer. J. Math. 95 333, (1973).

[4] H Widom, Eigenvalue distribution of nonselfadjoint Toeplitz matrices and the asymptotics of Toeplitz determinants in the case of nonvanishing index, Operator Theory: Adv. and Appl. 48 387, (1990).

[5] E Basor, K Morrison, The Fisher-Hartwig conjecture and Toeplitz eigenvalues, Linear Algebra and its Applications 202, 129 (1994).

[6] R Beam, R Warming, The asymptotic spectrum of banded Toeplitz and quasi-Toeplitz matrices, Presented in the 14th Biennial Conference on Numerical Analysis, Dundee, Scotland (1991).

[7] B M McCoy, T T Wu, The two-dimensional Ising model, Harvard University Press, Princeton (1973).

[8] A Böttcher, B Silbermann, Analysis of Toeplitz operators, 2nd ed, Springer, Berlin (2006).

[9] M E Fisher, R E Hartwig, Toeplitz determinants, some applications, theorems and conjectures, Adv. Chem. Phys. 15, 333 (1968).

[10] M E Fisher, R E Hartwig, Asymptotic behavior of Toeplitz matrices and determinants, Arch. Rat. Mech. Anal. 32, 190 (1969).

[11] http://en.wikipedia.org/wiki/ Wiener $\%$ E2\%80\%93Hopf_method 